\title{
Proteolipid protein 2 is associated with melanoma metastasis
}

\author{
YOSHIKO SONODA, MASAKO WARITA, TAKAHARU SUZUKI, HIROKI OZAWA \\ YUSUKE FUKUDA, MEGUMI FUNAKOSHI-TAGO and TADASHI KASAHARA
}

Faculty of Pharmacy, Keio University, Shibakoen 1-5-30, Minato-ku, Tokyo 105-8512, Japan

Received July 28, 2009; Accepted September 14, 2009

DOI: 10.3892/or_00000645

\begin{abstract}
The metastasis of malignant tumor cells from the primary tumor to distant sites in the body is a complex process. To identify genes that may be essential for metastasis, we established poorly metastatic mouse melanoma cells, namely Y925F-mutated FAK-transfected cells (Y925F cells), from the highly metastatic mouse melanoma cell line B16F10, and performed expression analyses. The expression of phospholipid protein 2 (PLP2) was markedly down-regulated in the Y925F cells. To elucidate the function of PLP2, we established melanoma cells overexpressing PLP2. We found that PLP2 enhanced proliferation, adhesion, invasion, and MMP-2 secretion in vitro, and tumor metastasis in vivo. These results suggest that PLP2 aids metastasis. Furthermore, we showed that PLP2 binds specifically to PI3K, thus activating Akt.
\end{abstract}

\section{Introduction}

The metastasis of malignant tumor cells from the primary tumor to distant sites in the body is a complex process (1). To study the mechanisms of metastasis, in vivo systems such as experimental metastasis models have been developed (2). Using these experimental models, the adhesion, proliferation, migration, and invasion of metastatic tumor cells have been revealed to play important roles. Results of experimental assays suggest proliferation to be a rate-limiting step during metastasis (3). Further, protein expression associated with proliferation, angiogenesis, adhesion, motility and invasion is correlated with metastasis (3). Signal transduction pathways activated by adhesion primarily involve focal adhesion kinase (FAK), p130Cas, and Paxillin. FAK mediates many cellular processes, including survival, migration, and invasion (4).

Previously, we reported that Y925F-FAK-transfected (Y925F) melanoma cells had impared metastatic ablity as compared with parental B16F10 cells or vector-transfected (Vect) cells (5). Metastatic genes can therefore be identified through microarray analyses by comparing the gene expression profiles of the highly metastatic Vect cells with those of the

Correspondence to: Dr Yoshiko Sonoda, Faculty of Pharmacy, Keio University, Shibakoen 1-5-30, Minato-ku, Tokyo 105-8512, Japan

E-mail: sonoda-ys@pha.keio.ac.jp

Key words: melanoma, metastasis, FAK, PLP2, PI3K, Akt poorly metastatic Y925F cells. Low levels of proteolipid protein 2 (PLP2) were found in Y925F cells following microarray analysis. In this study, we report that PLP2, a four-transmembrane protein, contributes to the metastasis of melanoma cells by interacting with a factor in the tissue or tumor microenvironment. Using biochemical methods, we identified the PI3K, ubiquitously expressed protein, as an associate of PLP2, which activates Akt, and passively enhances the MMP-2 secretion and the metastatic function of PLP2.

\section{Materials and methods}

Cell culture. The melanoma cell line B16F10 (6) was obtained from Professor Yoshikazu Sugimoto of Keio University and maintained in Dulbecco's modified Eagle's medium (DMEM) supplemented with $5 \%$ calf serum (CS).

Gene expression analysis using GeneChip. A GeneChip analysis of empty Vect-transfected B16F10 cells and Y925FFAK-transfected B16F10 was performed using oligonucleotide arrays (GeneChip AceGene 30K-1, containing 30,000 mouse genes). Briefly, Cy3- or Cy5-labeled cDNAs generated from these cells were hybridized to the DNA chips, and scanned with a Chip Reader (Vertek, Canada). The scanned images were then processed using the MicroArray Suite (Scanalytic, USA). Data were normalized by the median of intensity of a housekeeping gene (actin).

Immunoblotting and immunoprecipitation. Immunoblotting was performed using anti-PLP2, anti-PI3K, anti-phosphoAkt, Akt, and anti-phosphotyrosine, and immunoprecipitation was performed as described previously $(7,8)$.

Animals. C57BL/6J mice (6-8-week-old) were obtained from Nippon Charles River (Tokyo, Japan). Throughout the experiments, the mice were kept in plastic cages (at $21 \pm 2^{\circ} \mathrm{C}$ ) with free access to pellet food and water and on a 12-h lightdark cycle. Animal welfare and experimental procedures were performed strictly in accordance with the Guide for the Care and Use of Laboratory Animals and the related ethics regulations of our university. All efforts were made to minimize the animals' suffering and to reduce the number of animals used.

Plasmid preparation and transfection. The mouse PLP2 gene coding region was amplified from cDNA derived from 
melanoma B16F10 cells, using as a sense primer 5'-ATGGC GGATTCTGAGCCAGAA-3' and antisense primer 5'-TCA CGGGCCATCAGTGGGGT-3'. This coding region was ligated into a TA cloning vector. Next, the fragment restricted by EcoR1 was ligated into the vector pcDNA3.1 (Invitrogen, Inc., Carlsbad, CA). Clones containing the PLP2 insert were sequenced and a $100 \%$ matching clone was obtained. A plasmid for PLP2 was prepared and $350 \mathrm{ng}$ of plasmid was transfected into B16F10 cells using Lipofectamine Plus reagent (Invitrogen Life Technologies) following the manufacturer's protocol. The medium was replaced with normal growth medium after $24 \mathrm{~h}$. Further, G418 $(600 \mu \mathrm{g} / \mathrm{ml})$ was added to the growth medium $24 \mathrm{~h}$ after plating. Colonies were selected and screened by Western blotting in order to identify the PLP2-overexpressing cells. Cells transfected with empty pcDNA3.1 vectors served as controls.

Production of specific antiserum. Keyhole limpet haemocyanin (KLH)-conjugated peptides (70-84, CDLHSKISFINWPWT, 135-152, CPLKQQRHTAAPTDPTDGP) were used to immunize a Japanese White rabbit. Serum of the immunized rabbit was then passed over a protein A-Sepharose column and the IgG fraction was eluted and used in Western blotting and immunocytolocalization in B16F10 cells and PLP2-transfected cells.

Cell proliferation. Cell proliferation was evaluated by plating $2 \times 10^{3}$ cells in 96-wells containing growth medium and assayed by MTT. For the MTT assay, $10 \mu 1$ of MTT ( $5 \mu \mathrm{g} / \mu 1$ in PBS) was added to each well and cells were incubated at $37^{\circ} \mathrm{C}$ in a $\mathrm{CO}_{2}$ incubator. After $3 \mathrm{~h}$, cells were lysed using isopropanol- $\mathrm{HCl}$ and absorbance was measured at $570 \mathrm{~nm}$. All cell growth experiments were repeated at least three times.

Quantification of gene expression by real-time quantitative $P C R$. RNA was prepared from cells using a Quiagen Mini RNA-easy kit (Tokyo, Japan). Reverse transcription was performed from $1 \mu \mathrm{g}$ of RNA in a $20-\mu 1$ reaction mixture according to the manufacturer's instructions (Takara RNA PCR kit). PCR amplification and analysis were achieved using real-time PCR (Prism 7000, Applied Biosystems, CA). Primer sequences used were as follows: PLP2 sense, 5'-GT GTGACCTGCACTCCAAGA-3'; antisense, 5'-TCAGTGG GAGCTGCTGTATG-3'. GAPDH sense, 5'-ACTCCACTC ACGGCAAATTC-3'; antisense, 5'-CCTTCCACAATGCCA AAGTT-3'.

Cell adhesion assay. Ninety-six-well flat-bottomed plates (CytoMatrix screen kit, Chemicon, ECM205) coated with fibronectin, vitronectin, laminin, and collagen I were used. The cells were detached from their culture plates by dissociation buffer, seeded at a density of $6 \times 10^{4}$ cells/well in $100 \mu 1$ of medium, and incubated at $37^{\circ} \mathrm{C}$ for $2 \mathrm{~h}$. The assay was terminated by washing the plates with PBS to remove unbound cells. The cells that remained attached to the plates were fixed and stained with $0.5 \%$ crystal violet $/ 2 \% \mathrm{EtOH} / 0.1 \mathrm{M}$ borate buffer ( $\mathrm{pH}$ 9.0). After washing with PBS, $100 \mu \mathrm{l}$ of solubilization buffer (1:1 mixture of $0.1 \mathrm{M} \mathrm{NaH}_{2} \mathrm{PO}_{4}, \mathrm{pH} 4.5$ and $\mathrm{EtOH}$ ) was added to the plates and measurements were taken with an ELISA reader. The data were expressed as the mean absorbance of triplicate wells \pm SD.

Migration. Haptotaxis was assayed in triplicate using modified Boyden chambers, cell culture inserts (Falcon) with a polyethylene terephthalate membrane $(8-\mu \mathrm{m}$ pore size, $13-\mathrm{mm}$ diameter). Filters were precoated on the lower side with a $5 \mu \mathrm{g} / \mathrm{ml}$ fibronectin solution as described previously (5). After drying, the filters were placed in the lower chamber containing $1 \%$ CS-DMEM. Next, $1 \times 10^{5}$ cells were added to the upper chamber supplemented with $1 \%$ CS-DMEM and incubated at $37^{\circ} \mathrm{C}$ for $24 \mathrm{~h}$. The cells that had migrated to the underside of the insert membranes were stained with Giemsa and counted. Five fields per insert were scored, and the treatment was performed in duplicate.

Matrigel invasion assay. In vitro invasion assays were performed as described previously with some modifications (5). A Matrigel invasion chamber (Becton-Dickinson) was used. A chemoattractant, the conditioned medium of fibroblasts, was added to the lower chamber to induce invasion of cells through the Matrigel. Cells were added ( $1 \times 10^{5} /$ well) to the inner chamber of a cell culture insert and incubated at $37^{\circ} \mathrm{C}$ for $72 \mathrm{~h}$. In order to quantify this invasion, the filters were fixed in $70 \%$ ethanol for $30 \mathrm{~min}$ and stained with Giemsa. Non-invading cells were removed from the upper surface of the Matrigel by rubbing gently with a cotton-tipped applicator. Cells that had passed through the membrane were counted in 5 random microscopic fields of the lower filter surface.

Gelatin zymography. Cells (about $70-80 \%$ confluent) were washed, replenished with serum-free DMEM, and incubated for $24 \mathrm{~h}$. Serum-free conditioned medium was mixed with SDS buffer without heating or reduction and applied to $10 \%$ polyacrylamide gels containing $1 \mathrm{mg} / \mathrm{ml}$ of gelatin. After electrophoresis, gels were washed in $2.5 \%(\mathrm{v} / \mathrm{v})$ Triton X-100 for $2 \mathrm{~h}$ at room temperature to remove SDS, rinsed with water, and incubated in protease buffer $(50 \mathrm{mM}$ Tris- $\mathrm{HCl}, \mathrm{pH} 7.5$, $5 \mathrm{mM} \mathrm{CaCl}_{2}, 1 \mathrm{mM} \mathrm{ZnCl}$, and $0.1 \mathrm{mM} \mathrm{NaN}_{3}$ ) for $40 \mathrm{~h}$ at $37^{\circ} \mathrm{C}$. Gels were stained with $0.5 \%$ (deletion of $10 \%$ ) Coomassie Blue R-250 in 10\% 2-propanol and $10 \%$ acetic acid and destained. Protease activity was visualized as clear bands.

Tail vein metastasis. C57BL/6J mice were injected intravenously via the tail with $2.5 \times 10^{5}$ cells in $0.2 \mathrm{ml}$ of PBS. After 14 days, the mice were euthanized, and their lungs were resected and photographed. The metastatic nodules on the lung surface were counted macroscopically.

Statistical analysis. Data were expressed as the mean \pm SD. Results of in vitro experiments were analyzed with the Student's t-test. For in vivo experiments, the MannWhitney U test was used for comparing two groups. Values of $p<0.05$ and $p<0.01$ were considered statistically significant differences.

\section{Results}

PLP2 expression is down-regulated in poorly metastatic melanoma cell lines. Previously, when $2.5 \times 10^{5}$ cells from 
(A)

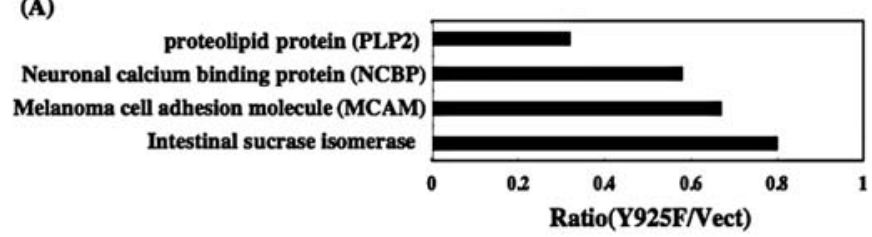

(B)

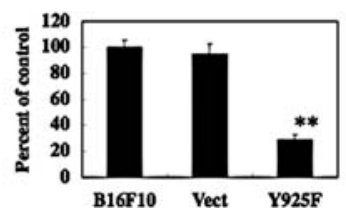

(C)

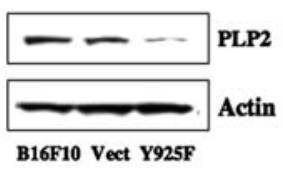

Figure 1. Down-regulated gene expression of PLP2 in Y925F cells compared with Vect cells. (A) Down-regulated gene in 925 cells compared with Vect cells as determined by a microarray analysis. Competitive hybridization was performed in Y925F cells and Vect cells. The RNA expression levels of genes were calculated by the ratio of the fluorescence intensity using microarray, and the values were corrected using the value for actin transcript as the internal control. (B) Relative levels of PLP2 mRNA in 925 cells. A real-time quantitative PCR analysis of PLP2 was performed as described in Materials and methods. Results are the mean for three experiments and expressed as a percentage of the control (Vect cells). ${ }^{* *} \mathrm{P}<0.01$ indicates values significantly different from the control (Vect cells). (C) Western blot analysis of PLP2 in 925 cells. Proteins from whole-cell lysates were separated using $12 \%$ SDS-PAGE followed by electroblotting onto nitrocellulose membranes. The Western blots were probed with either anti-PLP2 or anti-actin.

each cell line were injected intravenously into $\mathrm{C} 57 \mathrm{BL} / 6 \mathrm{j}$ mice, the Y925F cells produced fewer lung metastases than the parental or Vect cells at 2 weeks after injection (5). Expression levels of genes in tumors derived from the poorly metastatic variants and the highly metastatic Vect cells were compared by using ACE gene arrays and MicroArray Suite software. The PLP2 gene was significantly down-regulated, while the genes encoding neuronal calcium-binding protein (NCBP), melanoma cell adhesion molecule (MCAM), and intestinal sucrase-isomerase were slightly down-regulated in samples from Y925F cells (Fig. 1A). We confirmed by realtime PCR that PLP2 mRNA expression is down-regulated in Y925F cells (-1/5-fold, Fig. 1B). We focused on PLP2, whose gene expression was most decreased. To determine whether PLP2 is also affected at the protein level in the samples from the poorly metastatic Y925F cells, we prepared an antibody against the C-terminus of PLP2 (denoted anti-PLP2). This antibody specifically recognized a band of $17 \mathrm{kDa}$ in total lysate from cells expressing PLP2. This proves that the expression of PLP2 in Y925F cells is down-regulated (Fig. 1C).

Establishment of PLP2-transfected cells. To investigate the role of the PLP2 protein in aiding metastasis, melanoma B16F10 cells were transfected with an empty vector or PLP2 expression vector. Stable clones from each transfectant were obtained and designated as Vect cells (empty vector-transfected cells), or as PLP2-1, and PLP2-2 cells (PLP2-transfected cells). Analyses by Western blotting indicated that the PLP2 protein was expressed to a higher degree in the PLP2 cells than B16F10 or Vect cells (Fig. 2A). The overall expression of PLP2 was increased $~ 3-5$-fold in PLP2-1 and PLP2-2 cells as compared with Vect cells. Immunostaining with anti-PLP2 revealed PLP2 to be located at membranes (Fig. 2B). Cell proliferation influences metastasis (9). The PLP2 cells were analyzed in order to determine whether proliferation in these cells was affected. The PLP2 cells displayed enhanced proliferation as compared with the Vect cells (Fig. $2 \mathrm{C}$ ). As both clones displayed identical behavior, only data for 1 clone is shown.

Stable expression of PLP2 enhances adhesion, motility, invasion, and MMP-2 activation. To determine whether
(A)

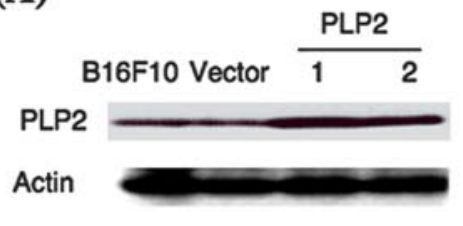

(C)

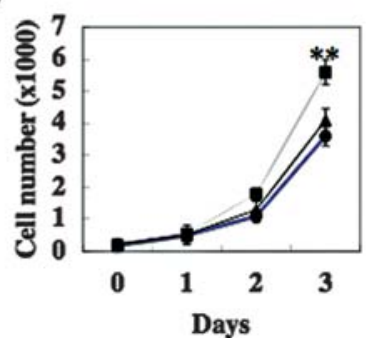

(B)
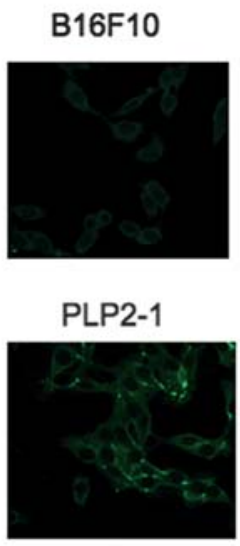

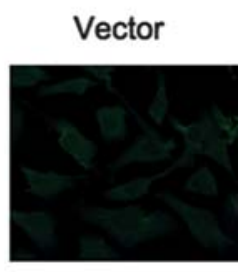

PLP2-2

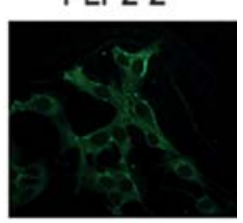

Figure 2. Characterization of transfected PLP2 cells. (A) Western blot analysis of PLP2 and actin in PLP2-transfected cells, clones 1 and 2. PLP2 immunoreactivity was markedly detected in lysates of PLP2-overexpressing B16F10 cells. Thirty micrograms of total protein isolated from cells was separated by $12 \%$ SDS-PAGE and transferred to a membrane for Western blot analysis. (B) Immunostaining of PLP2 in parental, Vect-, and PLP2-transfected cells, clones 1 and 2. Parental, Vect-, and PLP2-transfected cells were fixed and stained for PLP2. (C) Proliferation assay. Overexpression of PLP2 enhanced the proliferation of B16F10 cells in vitro. Cell proliferation was assessed using the MTT assay as described in Materials and methods. Cells were seeded at $2 \times 10^{3}$ cells per well in 96-well plates and grown for 3 days. Experiments were done three times in triplicate. Representative results from one experiment are shown (mean \pm SD). $(\bullet$, B 16F10; $\boldsymbol{\Delta}$, Vecto; $\mathbf{\square}, \mathrm{PLP} 2) .{ }^{* *} \mathrm{P}<0.05$ indicate values significantly different from the control (Vect cells). 
(A)

(C)
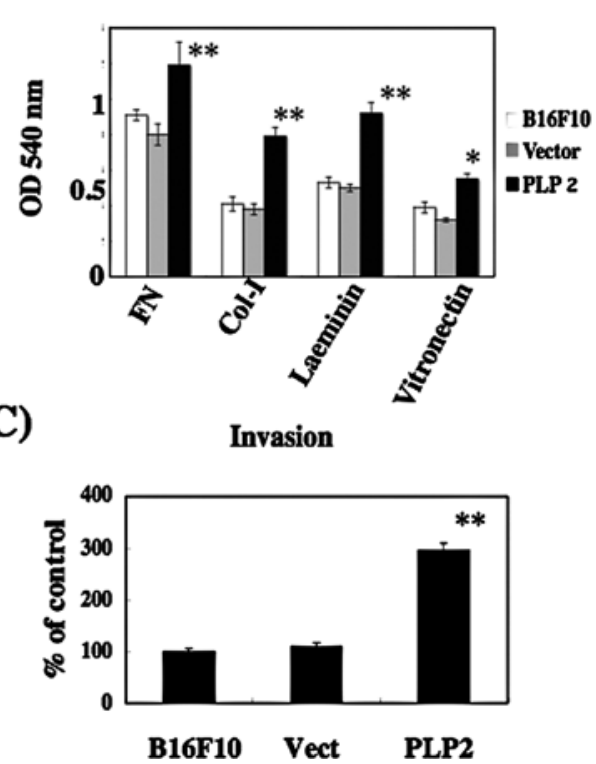

(B)

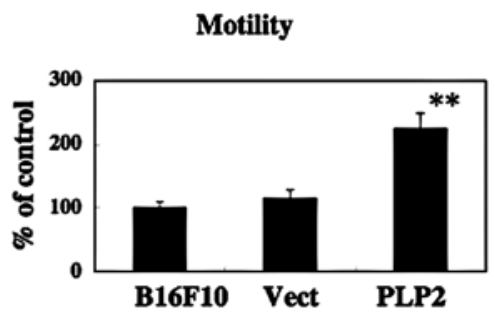

(D)

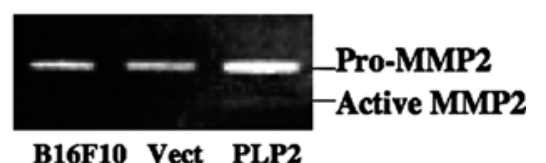

Figure 3. PLP2 enhanced adhesion, migration (haptotaxis), invasion, and MMP2 activation. (A) The adhesion of the parental melanoma cells or transfected melanoma cells to ECM (fibronectin, vitronectin, laminin, and collagen I) was determined as described in Materials and methods. The y-axis shows optical density following crystal violet staining of adhered cells. (B) The membranes of Boyden Chambers were coated with fibronectin and migration to the fibronectin (haptotaxis) was assayed. Furthermore, the conditioned medium of fibroblasts was added to the lower chamber of fibronectin-coated chambers. The $\mathrm{y}$-axis represents the average proportion of migrating cells ( $\%$ of control) in one field that penetrated the membrane to the lower chamber. (C) The membranes of Boyden Chambers were coated with matrigel and invasion was assayed. The conditioned medium of fibroblasts was added to the lower chamber. The $\mathrm{y}$-axis represents the average proportion of migrating cells (\% of control) in one field that penetrated the membrane to the lower chamber. ${ }^{*} \mathrm{P}<0.05,{ }^{* *} \mathrm{P}<0.01$ indicate values significantly different from the control (Vect cells). (D) Gelatin zymography of conditioned media from cells to detect MMP2. Cells were incubated in serum-free media for $24 \mathrm{~h}$ and conditioned media were collected for analysis.

(A)

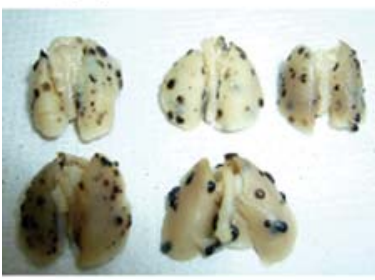

B16F10

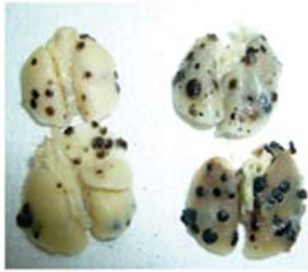

Vector

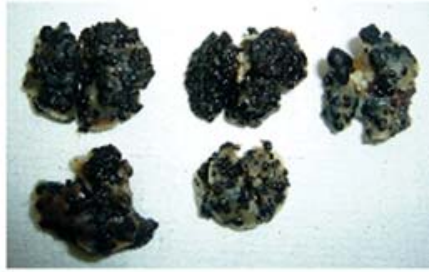

PLP2

(B)

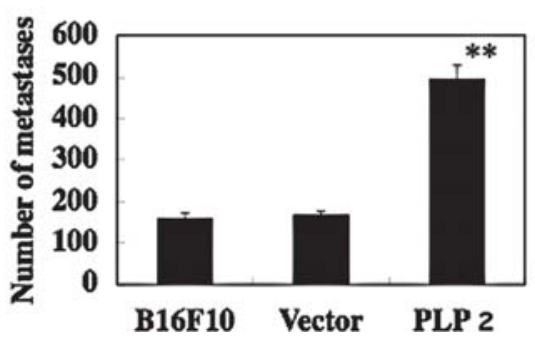

Figure 4. Effects of PLP2 on melanoma metastasis in vivo. Parental and transfected cells (2.5x105/mouse) were intravenously injected into C57BL/ 6J mice via the tail. After 14 days, lungs were resected and analyzed for metastasis. (A) Representative photographs of lungs. (B) Quantitative evaluation of macroscopically detectable lung metastasis. Data are means \pm SD for five mice. ${ }^{* *} \mathrm{P}<0.01$ indicates values significantly different from the control (Vect cells).

adhesion was affected in PLP2 cells, we tested attachment to the ECM (fibronectin, vitronectin, laminin, and collagen I) using a cytomatrix kit. Under identical experimental conditions, the PLP2 cells showed potentiated adhesion to fibronectin, vitronectin, and collagen I as compared with the parental and Vect cells during $2 \mathrm{~h}$ of incubation. As both clones displayed 
(A)

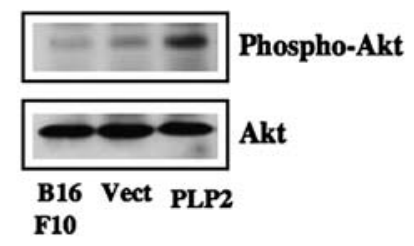

(B)

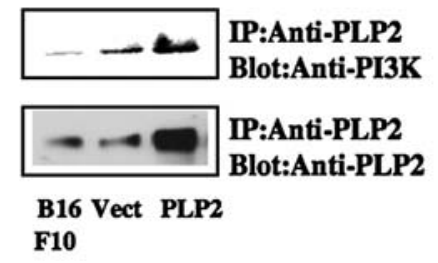

(C)

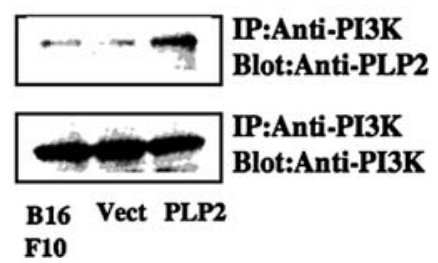

Figure 5. Detection of the association of PI3K with PLP2 and the Akt activation in PLP2 cells. (A) Lysates were prepared and blotted with anti-phosphoAkt and anti-Akt. (B). Lysates were prepared and immunoprecipitated by anti-PLP2, and blotted with anti-PI3K or anti-PLP2. (C) Lysates were prepared and immunoprecipitated by anti-PI3K, and blotted with anti-PLP2 or anti-PI3K.

identical behavior, only data for 1 clone is shown (Fig. 3A). These results suggest that PLP2 is vital to the adhesive capability of melanoma cells.

To determine the involvement of the PLP2 (deletion of cells) in determining motility in the $\mathrm{B} 16 \mathrm{~F} 10$ cells, a haptotaxis assay was performed in which migration to fibronectin was evaluated using a modified Boyden chamber (Fig. 3B). The haptotaxis of the PLP2 cells was significantly potentiated as compared with that of the parental and Vect cells ( $>180 \%$ of that of B16F10 and Vect). These results demonstrate that the PLP2 cells have a positive influence on haptotaxis.

In addition, we performed a matrigel invasion assay with a fibroblast-conditioned medium that contained a growth factor in the lower chamber, which evaluated the ability of these cells to respond to a growth factor. Interestingly, the invasive capacity of the PLP2 cells was found to be enhanced (Fig. 3C). These results suggest that PLP2 is required to elicit a response to growth factor receptor-mediated signaling. However, further study will be required to explain the function of PLP2 in growth factor receptor-mediated signaling.

As a crucial step in the invasion involves the degradation of extracellular matrix (ECM) components, which allows cells to efficiently traverse basement membranes, we examined MMP activity. Gelatin zymography of serum-free conditioned medium revealed that parental cells secreted MMP-2, and PLP2 cells increased the level of pro- and activated MMP-2 (Fig. 3D). However, MMP-9 secretion was not detected in parental, vect, or PLP2 cells.

Induction of the Akt activation and PI3K-PLP2 association in PLP2 cells. To examine the effect of the PLP2 cells on metastasis, the mechanism of signal transduction was studied. We first found Akt to be phosphorylated in the PLP2 cells (Fig. 5A). As Akt was activated in the downstream to PI3K, we hypothesized that PLP2 was associated with PI3K and the activation of Akt. As expected, PLP2 was found to be associated with PI3K. This association was clearly evident in the PLP2 cells, but weak in the Vect cells (Fig. 5B and C)
These results indicate that PLP2 potentiates the activation of Akt via PI3K.

Metastasis is enhanced in PLP2 cells. Experiments were performed in vivo since the Matrigel invasion assay does not fully reproduce the multiple steps of metastasis. PLP2 cells $\left(2.5 \times 10^{5}\right)$ were injected intravenously, through the tail, and lung metastasis was evaluated after 2 weeks (Fig. 4). The animals injected with parental B16F10 or Vect cells had multiple tumor nodules on their lungs. Metastasis was enhanced $296 \%$ in the mice injected with PLP2 cells $(167 \pm 10$ vs. $495 \pm 33$ nodules, $\mathrm{p}<0.01)$ compared with those injected with Vector cells.

\section{Discussion}

We found that PLP2 expression was down-regulated in the poorly metastatic melanoma cell lines, the Y925F-FAKtransfected cells, as compared with the highly metastatic parental cells, B16F10, and empty vector-transfected cells. We also found that PLP2 played important roles in enhancing metastasis in our experimental model: overexpression of PLP2 led to a progression of the metastasis of melanoma cells. The increased metastasis might partially depend on differences in the proliferation of cells, since the proliferation rate of PLP2 cells was higher than that of the parental, or Vect cells. Further, we observed that the PLP2 cells showed adhesion, migration and invasion, and secretion of MMP-2 in vitro. Degradation of the ECM by MMP is required for invasion. MMPs are a family of structurally related $\mathrm{Zn}^{2+}$ dependent metalloproteinases. Studies have demonstrated that MMP-2 or -9 plays a key role in invasion. The PI3K-Akt signaling pathway is one of the most important downstream targets of receptor tyrosine kinases. Upon stimulation, the p85 subunit of PI3K is recruited to the membrane and phosphorylated by kinases. The activated PI3K stimulates Akt and various downstream effectors controlling cellular proliferation, migration, and invasion. There are many reports that 
the PI3K-Akt signaling pathway is involved in cell survival, migration, and invasion. Furthermore, the PI3K-Akt pathway is assumed to have a variety of functions in cell biology. For example, Akt converted radial growth to vertical growth in melanoma cells (10). Recently, PI3K-Akt signaling has been reported to be involved in the hepatocyte growth factorinduced migration of uveal melanoma cells (11).

PLP2 was reported to be associated with BAP31 and resistant to Fas-induced apoptosis (12). BAP31 is emerging as a putative chaperon/quality control factor that regulates the fate of integral membrane proteins of the ER membrane. In this study, the association of PLP2 and BAP31 was observed (data not shown). The antiapoptotic function of PLP2 needs to be studied further.

PLP2 was associated with CCR1 and stimulated migration in CCR1-expressing HOS cells (13). In the present study, no CCR1 was found in these cells (data not shown). The association of chemokine receptors with PLP2 function needs to be studied further. PLP2 has 4 potential membranespanning domains $(12,14)$. PLP2 exhibits similarities with subunit $\mathrm{c}$ of the proton-containing vascuolar ATPase (14). Immunofluorescence analysis revealed PLP2 to be present predominantly on the cell surface. This suggests that PLP2 functions on membranes. In conclusion, our results demonstrated that PLP2 promoted metastasis in melanoma B16F10 cells in vivo, and provide evidence that PLP2 is a potential therapeutic target.

\section{Acknowledgements}

We thank to Kumi Ando, Tomoyuki Oshio, Tomoko Kawaguchi, and Nagisa Ishiwata for their technical assistance. This study was supported in part by a grant (No. 20590069) from the Ministry of Education, Culture, Sports, Science and Technology of Japan.

\section{References}

1. Fidler IJ: The pathogenesis of cancer metastasis: the 'seed and soil' hypothesis revisited. Nat Rev Cancer 3: 453-458, 2003.

2. MacDonald IC, Groom AC and Chambers AF: Cancer spread and micrometastasis development: quantitative approaches for in vivo models. Bioessays 24: 885-893, 2002.
3. Hendrix MJ, Seftor EA, Seftor RE, Kasemeier-Kulesa J, Kulesa PM and Postovit LM: Reprogramming metastatic tumour cells with embryonic microenvironments. Nat Rev Cancer 7: 246-255, 2007.

4. Hanks SK, Ryzhova L, Shin NY and Brábek J: Focal adhesion kinase signaling activities and their implications in the control of cell survival and motility. Front Biosci 8: 982-996, 2003.

5. Kaneda T, Sonoda Y, Ando K, Suzuki T, Sasaki Y, Oshio T, Tago M and Kasahara T: Mutation of Y925F in focal adhesion kinase (FAK) suppresses melanoma cell proliferation and metastasis. Cancer Lett 270: 354-361, 2008.

6. Raz A, Bucana C, McLellan W and Fidler IJ: Distribution of

lung colonising potential. Nature 284: 363-364, 1980.

7. Sonoda Y, Watanabe S, Matsumoto Y, Aizu-Yokota E and Kasahara T: FAK is the upstream signal protein of the phosphatidylinositol 3-kinase-Akt survival pathway in hydrogen peroxide-induced apoptosis of a human glioblastoma cell line. J Biol Chem 274: 10566-10570, 1999.

8. Sonoda Y, Matsumoto Y, Funakoshi M, Yamamoto D, Hanks SK and Kasahara T: Anti-apoptosis of focal adhesion kinase (FAK). Induction of inhibitor-of-apoptosis proteins and apoptosis suppression by the overexpression of FAK in a human leukemic cell line, HL-60. J Biol Chem 275: 16309-16315, 2000.

9. Danen EHJ and Yamada KM: Fibronectin, integrins, and growth control. J Cell Physiol 189: 1-13, 2001.

10. Govindarajan B, Sligh JE, Vincent BJ, Li M, Canter JA, Nickoloff BJ, Rodenburg RJ, Smeitink JA, Oberley L, Zhang Y, Slingerland J, Arnold RS, Lambeth JD, Cohen C, Hilenski L, Griendling K, Martínez-Diez M, Cuezva JM and Arbiser JL: Overexpression of Akt converts radial growth melanoma to vertical growth melanoma. J Clin Invest 117: 719-729, 2007.

11. Ye M, Hu D, Tu L, Zhou X, Lu F, Wen B, Wu W, Lin Y, Zhou $\mathrm{Z}$ and Qu J: Involvement of PI3K/Akt signaling pathway in hepatocyte growth factor-induced migration of uveal melanoma cells. Invest Ophthalmol Vis Sci 49: 497-504, 2008.

12. Wang B, Nguyen M, Breckenridge DG, Stojanovic M, Clemons PA, Kuppig S and Shore GC: Unleaved BAP31 in association with $\mathrm{A} 4$ protein at the endoplasmic reticulum is an inhibitor of Fas -initiated release of cytochrome $\mathrm{c}$ from mitochondoria. J Biol Chem 278: 1446-1448, 2003.

13. Lee SM, Shin H, Jang SW, Shim JJ, Song IS, Son KN, Hwang J, Shin YH, Kim HH, Lee CK, Ko J, Na DS, Kwon BS and Kim J: PLP2/A4 interacts with CCR1 and stimulates migration of CCR1-expressing HOS cells. Biochem Biophys Res Commun 324: 768-772, 2004.

14. Breitwieser GE, McLenithan JC, Cortese JF, Shields JM, Oliva MM, Majewski JL, Machamer CE and Yang VW: Colonic epithelium-enriched protein A4 is a proteolipid that exhibits ion channel characteristics. Am J Physiol 272: C957C965, 1997. 